\title{
Model for the fine structure of zero field steps in long Josephson tunnel junctions and its comparison with experiment
}

Barbara, Paola; Monaco, R.; Ustinov, A. V.

Published in:

Journal of Applied Physics

Link to article, DOI:

$10.1063 / 1.360834$

Publication date:

1996

Document Version

Publisher's PDF, also known as Version of record

Link back to DTU Orbit

Citation (APA):

Barbara, P., Monaco, R., \& Ustinov, A. V. (1996). Model for the fine structure of zero field steps in long Josephson tunnel junctions and its comparison with experiment. Journal of Applied Physics, 79(1), 327-333. https://doi.org/10.1063/1.360834

\section{General rights}

Copyright and moral rights for the publications made accessible in the public portal are retained by the authors and/or other copyright owners and it is a condition of accessing publications that users recognise and abide by the legal requirements associated with these rights.

- Users may download and print one copy of any publication from the public portal for the purpose of private study or research.

- You may not further distribute the material or use it for any profit-making activity or commercial gain

- You may freely distribute the URL identifying the publication in the public portal 


\title{
Model for the fine structure of zero field steps in long Josephson tunnel junctions and its comparison with experiment
}

\author{
P. Barbara ${ }^{a)}$ \\ Physics Department, Technical University of Denmark, DK-2800 Lyngby, Denmark \\ R. Monaco a),b) \\ Instituto di Cibernetica del C.N.R., I-80072 Arco Felice (NA), Italy
}

\author{
A. V. Ustinov \\ Institute of Thin Films and Ion Technology, Research Center (KFA), D-52425 Jülich, Germany
}

(Received 9 June 1995; accepted for publication 7 September 1995)

\begin{abstract}
The single fluxon dynamics in the resonant regime has been investigated on high-quality low-loss $\mathrm{Nb} / \mathrm{Al}-\mathrm{AlO}_{x} / \mathrm{Nb}$ window Josephson tunnel junctions. A new model accounting for the fine structure of zero field steps in linear junctions is proposed. Depending on the fluxon velocity and on the junction losses, resonances may occur between the fluxon and the plasma waves corresponding to different wave numbers. This mechanism relies on a single proposition that the fluxon experiences an acceleration approaching the nonideal boundaries of the junction. We find good agreement between theory, numerical simulations, and experiments. The presence of the idle region has been proved to play a determinant role for the occurrence of the fine structure. (C) 1996 American Institute of Physics. [S0021-8979(95)03624-1]
\end{abstract}

\section{INTRODUCTION}

In this work we study the resonant oscillatory motion of fluxons (Josephson vortices, or solitons) in linear Josephson tunnel junctions. The shuttlelike fluxon motion is manifested by singularities in the current voltage characteristic of a long junction in absence of the external magnetic field. These singularities are called zero field steps (ZFSs). In particular, here we investigate the dynamics of a single fluxon, i.e., when the junction is biased on the first zero field step (ZFS1).

The velocity of the fluxon is determined by a balance between a driving force acting on the fluxon and the dissipative losses which occur during its motion. The driving force is proportional to the bias current density flowing across the junction; the losses are given by the normalized quasiparticle tunneling coefficient $\alpha$ and the losses in the superconducting electrodes determined by the surface loss coefficient $\beta$. Within a perturbational approach, a relativistic formula for the fluxon motion can be derived ${ }^{1}$ as

$$
\eta=\frac{4 \alpha p}{\pi}\left(1+\frac{\beta}{3 \alpha}\left(1+p^{2}\right)\right),
$$

where $\eta$ is the bias current normalized to the junction critical current $I_{c}, p=u \gamma(u), \gamma(u)=1 /\left(1-u^{2}\right)^{1 / 2}$, and $u$ is the average velocity of the fluxon normalized to the Swihart velocity $\bar{c}=c_{0} \sqrt{t / \epsilon \Lambda}$, the maximum velocity of electromagnetic wave propagation in a Josephson transmission line. Here $c_{0}$ $=1 / \sqrt{\epsilon_{0} \mu_{0}}$ is the light velocity in vacuum; $t, \epsilon$, and $\Lambda$ are the thickness of the tunnel barrier, its dielectric constant, and the magnetic thickness of the barrier, respectively. We recall that the average velocity of the fluxon is proportional to the $\mathrm{dc}$

\footnotetext{
${ }^{a)}$ Also with: Dipartimento di Fisica, Universitá di Salerno, I-84084 Baronissi (SA), Italy.

b)Electronic mail: roberto@vaxsa.csied.unisa.it
}

voltage across the junction according to the relation $V=\Phi_{0} \bar{c} / L u$ in which $\Phi_{0}=2.07 \times 10^{-15} \mathrm{~Wb}$ is the magnetic flux quantum and $L$ is the junction length.

Although Eq. (1) has been derived for infinitely long junctions, it quite accurately expresses the current-voltage relation for the ZFS1 branch on finite devices. In annular junctions, where a fluxon moves without reflections from the boundaries, the smooth profile predicted by Eq. (1) is in good accord with experimentally measured data. ${ }^{2}$ At the same time, data measured with low-loss linear junctions with open boundaries systematically show pronounced deviations from the form predicted by Eq. (1). These deviations are usually noted as fine structure of ZFSs. Generally, the smooth profile of the ZFS splits in a series of rather sharp resonances which occur at different voltages along the step. The first report on these structures dates back to 1981; they were observed on the $I-V$ characteristics of electrically long cross-type $\mathrm{Sn}$-oxide-Sn junctions. ${ }^{3}$ Later, fine structure was reported also in long in-line $\mathrm{Sn}$-oxide-Sn junctions. ${ }^{4}$ More recently, Monaco, Barbara, and Mygind, ${ }^{5}$ by measuring the frequency of the radiation emitted by intermediate-length overlap $\mathrm{Nb} / \mathrm{Al}-\mathrm{AlO}_{x} / \mathrm{Nb}$ junctions biased on the ZFS1, have reported accurate data on the fine structure, in particular on their temperature dependence.

A number of models have been proposed to explain the existence of the fine structures. Chang et al. ${ }^{4}$ related fine structure resonances to the interaction of $n$ fluxon oscillations with the $m$ th fractional cavity mode, assuming the response of the Josephson resonator to be linear: In this case, the resonant frequencies should be rational with respect to the characteristic frequency $f_{c}=\bar{c} /(2 L)$. Later, taking into account the nonlinear nature of a Josephson cavity, the fine structure was ascribed to the interaction of the traveling fluxon with small amplitude plasma oscillations with the frequency $f_{p}=(1 / 2 \pi) \sqrt{2 e I_{c} / \hbar C}$ (where $C$ is the junction ca- 


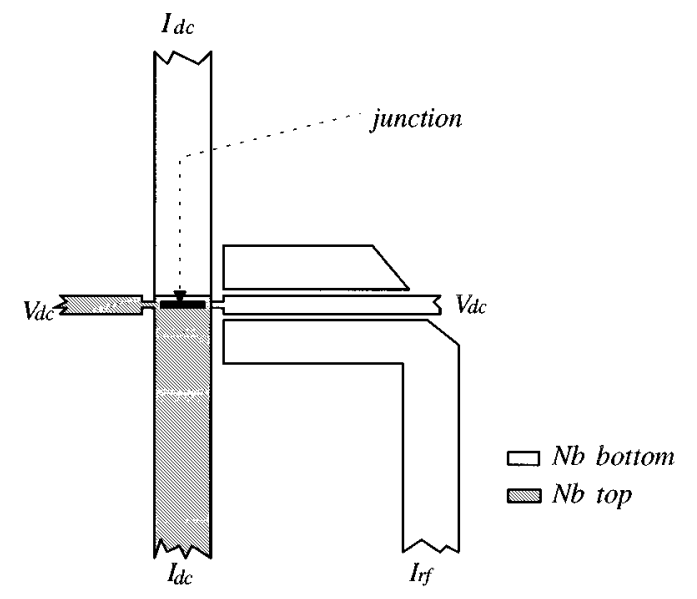

FIG. 1. Schematic of the sample geometry showing the dc and the rf leads.

pacitance and $I_{c}$ is its critical current): In this case, the resonant frequencies should occur at $f_{n}=f_{p} / n$. ${ }^{4,6}$

In a recent article $^{5}$ we have described the development of the fine structure steps at different temperatures and pointed out that the structure cannot be explained in terms of the previously suggested models. ${ }^{3,4,6}$ In this article we present more experimental data and propose a new model accounting for the fine structure of zero field steps. The case of the first zero field step (ZFS1) is mainly investigated, since on higher-order steps the fine structure can be ascribed to different arrangements of the fluxons in the barrier. ${ }^{7}$ When a fluxon gets reflected from the junction edges, in addition to having an antifluxon created, other excitations such as plasma waves are generated. Using a model by Golubov and Ustinov $^{8}$ proposed before for periodically inhomogeneous barriers, we show that, depending on the fluxon velocity, resonances may occur between the fluxon and the plasma waves corresponding to different wave numbers. For a long homogeneous junction such a radiation mechanism relies on a single proposition; that the boundaries of the long junction are not ideal and that the fluxon experiences an acceleration when it approaches the junction edges. We find good agreement between the theory and experiments made with highquality $\mathrm{Nb} / \mathrm{Al}-\mathrm{AlO}_{x} / \mathrm{Nb}$ window Josephson tunnel junctions.

This article is organized as follows. In Sec. II we describe the actual experiment and present the data. Their interpretation follows in Sec. III in the light of the simple theoretical model supported by numerical simulations. Finally, the conclusions are presented in Sec. IV.

\section{EXPERIMENTS}

The samples have been fabricated with the $\mathrm{Nb} / \mathrm{Al}-\mathrm{AlO}_{x} / \mathrm{Nb}$ trilayer technology at the University of Salerno. They consist of quasione-dimensional Josephson tunnel junctions in the overlap configuration, coupled to a microstrip line through a capacitive gap; a sketch of the geometry is shown in Fig. 1. The junctions were designed with characteristic frequencies within the $X$ band.

The deposition of the trilayers was made by rf magnetron sputtering, and the tunnel barriers were obtained by the
TABLE I. Electrical and geometrical parameters of the two samples. Data were measured at $T=4.2 \mathrm{~K}$.

\begin{tabular}{lcc}
\hline \multicolumn{1}{c}{ Sample } & $\mathrm{A}$ & $\mathrm{B}$ \\
\hline Length $L(\mu \mathrm{m})$ & 500 & 350 \\
Width $W(\mu \mathrm{m})$ & 20 & 20 \\
Critical current $I_{c}(\mathrm{~mA})$ & 1.15 & 1.0 \\
Critical current density $J_{c}\left(\mathrm{~A} / \mathrm{cm}^{2}\right)$ & 11.5 & 14.3 \\
Normal resistance $R_{n}(\Omega)$ & 1.4 & 1.3 \\
Subgap resistance $R_{\mathrm{sg}}$ at $2 \mathrm{mV}(\Omega)$ & 28 & 31 \\
Gap voltage $V_{g}(\mathrm{mV})$ & 2.75 & 2.60 \\
Quality factor $V_{m}(\mathrm{mV})$ & 32 & 31 \\
Magnetic thickness $\Lambda(\mathrm{nm})$ & 160 & 160 \\
Josephson length $\lambda_{J}(\mu \mathrm{m})$ & 120 & 108 \\
Normalized length $L / \lambda_{J}$ & 4.0 & 3.2 \\
Specific capacitance $\left(\mathrm{F} / \mathrm{m}^{2}\right)$ & 0.03 & 0.03 \\
\hline \hline
\end{tabular}

thermal oxidation of a thin aluminum overlayer. The trilayers were patterned with the geometry of the base electrode by reactive ion etching of the $\mathrm{Nb}$ layers and chemical etching of the $\mathrm{Al}$ layer. The area of the junctions was defined by the selective niobium anodization process, ${ }^{9}$ in which part of the top $\mathrm{Nb}$ layer in the region surrounding the junction is anodized. Finally, after a sputter cleaning of the top electrode surface, a $\mathrm{Nb}$ wiring film was deposited and patterned by lift-off. Details of the fabrication technique have been reported elsewhere. ${ }^{10}$ This fabrication process provides window junctions having a rather large area of the idle region (the surrounding $\mathrm{Nb}_{2} \mathrm{O}_{5}$ layer between the base electrode and the wiring film).

A larger number of samples has been measured. The characteristic parameters of two selected junctions discussed in this article are listed in Table I. All the electrical parameters have been calculated at $4.2 \mathrm{~K}$ ignoring the small effect due to the presence of the idle region surrounding the junction. The magnetic thickness $\Lambda$ was evaluated from the pattern in magnetic field of small junctions produced during the same fabrication process. The data in Table I show that the junctions A and B are good quality, intermediate length junctions; their characteristic resonance frequencies were within the expected microwave range.

In all the samples we have detected the radiation emitted at the fundamental frequency for different bias points of the ZFS1 in a broad range of temperatures. Due to the Josephson voltage-frequency relation, this type of measurement provides the most accurate determination of the ZFS1 $I-V$ profile. The rf circuit consisted of an $X$-band detection system. The $50 \Omega$ microstrip on the sample was connected with silver paint to the inner part of a coaxial cable. The emitted radiation was detected by a sensitive broadband microwave receiver and displayed on a spectrum analyzer. A series of microwave rejection filters and isolators, rf filtering, and passive electrical and magnetic shielding prevented unwanted signals and fields from interfering with the measurements. The detected power was weakly frequency dependent, but varied with the bias current; the frequency resolution was within $100 \mathrm{kHz}$. We could change the temperature by pumping on the helium bath and the pressure was stabilized with a cartesian manostat within 2 mbar. 


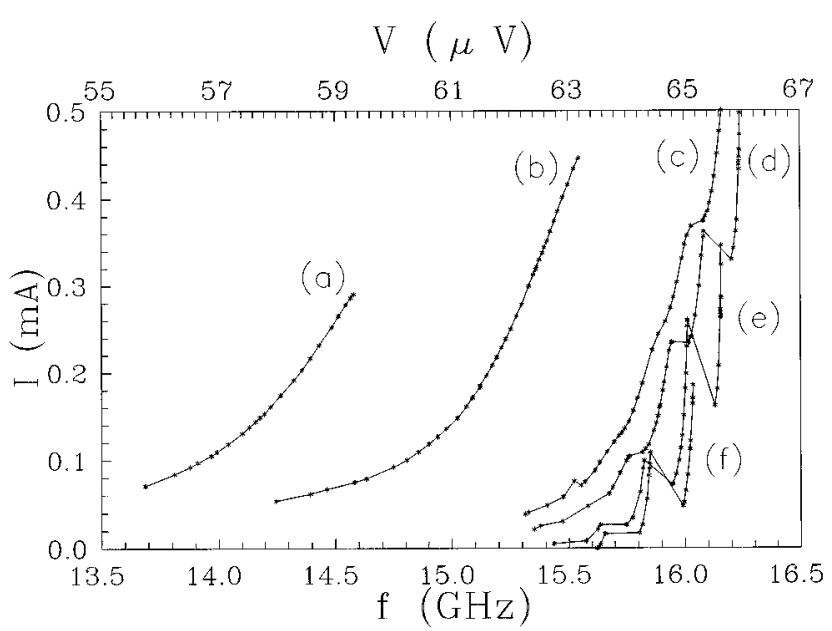

FIG. 2. Current-voltage characteristics ( $I-V$ curves) of the first zero field step (ZFS1) of sample B at different temperatures: (a) $8 \mathrm{~K}$; (b) $7 \mathrm{~K}$; (c) 4.2 $\mathrm{K}$; (d) $3.8 \mathrm{~K}$; (e) $3.0 \mathrm{~K}$; (f) $2.1 \mathrm{~K}$. The solid lines connecting the experimental points at each temperature are drawn as a guide to the eye.

The $I-V$ curves of the ZFS1 in sample B for different temperatures are shown in Fig. 2. Similar data for sample A have been reported in Ref. 5. The behavior of samples A and $B$ is qualitatively the same. One can clearly see the appearance of a fine structure in the curves corresponding to lower temperatures. The frequencies of the resonances are almost, but not exactly, equally spaced. In some cases the frequency jumps were as large as $500 \mathrm{MHz}$ (corresponding to about 1 $\mu \mathrm{V}$ in terms of voltage jump). The $I-V$ curves in Fig. 1 show a very low differential resistance $R_{d}=d V / d I$ at lower temperatures. As a typical value, we report the differential resistance of this sample at the top part of the ZFS1 $(I=0.29$ $\mathrm{mA}$ ) at $4.2 \mathrm{~K}$ as low as $0.9 \mathrm{~m} \Omega$. According to the theory described in Ref. 11, the linewidth of the radiation emitted by the junction in this state can be estimated to be less than $0.5 \mathrm{kHz}$. This value is by a factor of 10 lower than the linewidth data for $\mathrm{Nb}-\mathrm{Pb}$ junctions reported in the literature. ${ }^{11}$ The measurements of the quality factors $V_{m}$ (see Table I) confirm that the trilayer technology produces junctions with very low losses.

Besides its dependence on temperature and bias current, the frequency $f$ of the radiation emitted by the junction is sensitive to an external magnetic field $H$. We have measured the magnetic tuning of the radiation $d f / d H$ for several values of bias current and temperature. The measurements show a qualitative agreement with the results of the perturbation calculation outlined in Ref. 12 according to which intermediate length junctions should experience negative tuning $d f /$ $d H<0$ at the top of the step and positive tuning $d f / d H>0$ at the bottom. Our samples show a positive tuning for lowbias currents and small magnetic field. For higher bias points the tuning is smaller: At the top of the step a weak positive tuning can be measured only for temperature higher than 4.2 $\mathrm{K}$. This fact suggests that the step presents a premature switching point due to the fluxon instability at high speed which may impede the observation of other possible branches at higher voltages. This hypothesis of the premature

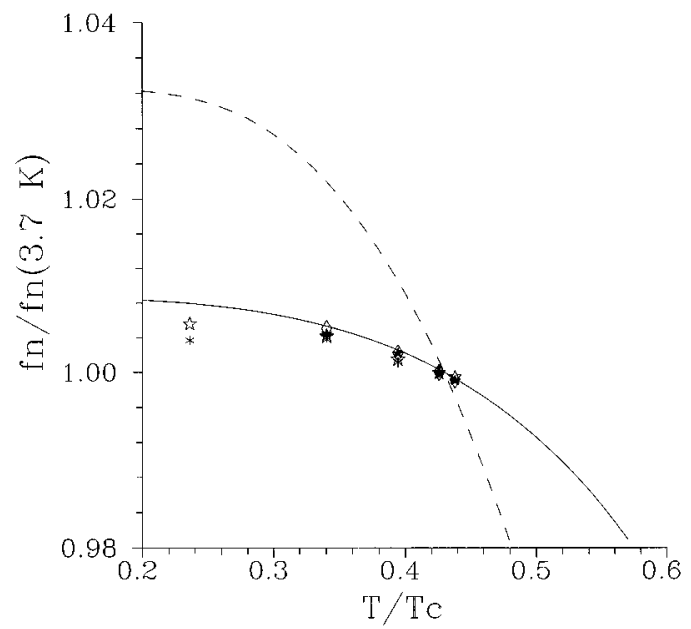

FIG. 3. Experimental values (points) of four fine structure resonance frequencies $f_{n}$ at different temperatures for sample B. Dashed line shows the temperature dependence of the plasma frequency $f_{p}$ [see Eq. (2)]. Solid line corresponds to the temperature dependence of the characteristic frequency $f_{c}$ [see Eq. (3)]. The frequencies are normalized to their values at $3.7 \mathrm{~K}$.

switching is strongly supported by the numerical simulation of Pagano et al. ${ }^{13}$ according to which the stability of the fluxon motion at high bias is drastically reduced when low $\alpha$ losses and large $\beta$ losses are present, as in our devices.

The measurements reported in Fig. 1 show that the asymptotic voltage of the resonances depends on temperature. In Ref. 5 we have tentatively related frequencies $f_{n}$ corresponding to these resonances to a frequency $f^{*}$, very close to the plasma frequency, by $f_{n}=(\mathrm{n} / \mathrm{m}) f^{*}$ with arbitrary integers $n$ and $m$. The fit to the experimental data, although good, was probably due to the use of two fitting parameters; moreover, the numbers which come out were not physically convincing. We have further investigated this hypothesis by analyzing the temperature dependence of the resonances. We made comparison of the data with the theoretical temperature dependence of the plasma frequency $f_{p}$ and of the characteristic frequency $f_{c}$. The temperature dependencies of $f_{p}$ and $f_{c}$ are given by the relations

$$
\begin{aligned}
& f_{p}=f_{p 0}\left[I_{c}(T) / I_{c 0}\right]^{1 / 2}, \\
& f_{c}=f_{c 0}\left[1-\left(T / T_{c}\right)^{4}\right]^{1 / 4},
\end{aligned}
$$

where $I_{c}$ is the junction critical current and the index 0 indicates the value of the parameters at $T=0$. The corresponding curves are traced in Fig. 3 together with the experimental values of $f_{n}$ at different temperatures and for four different values of the index $n$. The temperature behavior of the resonances fits nicely with the curve corresponding to the characteristic frequency. This fact indicates that the resonances cannot simply be rationally related to the plasma frequency and are instead proportional to the Swihart velocity. Moreover, the models previously proposed for interaction between the traveling fluxon and the small amplitude oscillations at the plasma frequency, in the limit of zero bias and $k=0$, cannot be applied in our case. In fact, due to the very low losses of our samples, the approximation of small amplitude, 

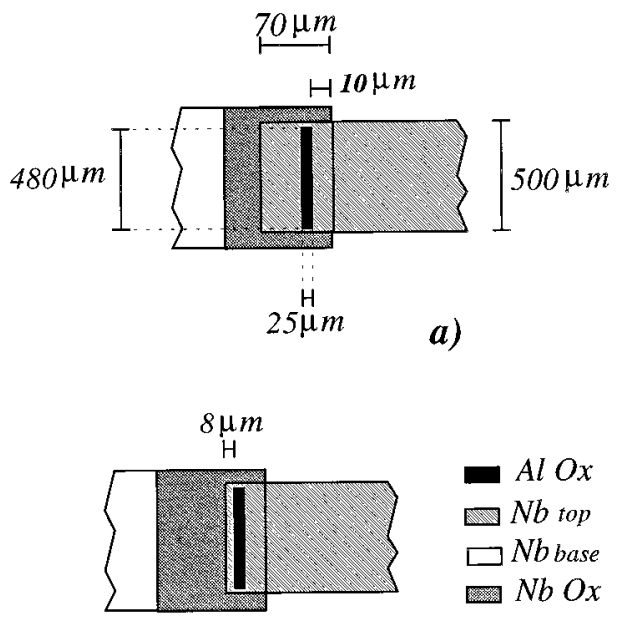

b)

FIG. 4. Geometry of the idle region for sample A (top view on the substrate plane): (a) before the reduction of the top electrode area; (b) after an asymmetric lateral reduction of the top electrode by about $30 \mu \mathrm{m}$.

bias-independent and $k=0$ plasma oscillation cannot be justified. In the new model which we propose in the following section these approximations are removed.

We would like to point out that the idle region surrounding the junction has also been found to be related to the appearance of fine structure on the ZFS1. ${ }^{14,15}$ In order to clarify this point, we have measured sample A before and after changing the dimensions of the idle region. By etching the wiring film, we have first symmetrically reduced the longitudinal dimension of the idle region from 600 to $500 \mu \mathrm{m}$. Since the junction length is $480 \mu \mathrm{m}$, after this modification the distance between the idle region boundary and the junction boundary was about $10 \mu \mathrm{m}$ as shown in Fig. 4(a). We measured the ZFS1 again [see Fig. 5(a)] and found that the fine structure appeared practically unchanged: The voltage positions of the branches were the same (within the experimental error of about $0.2 \mu \mathrm{V}$ ). Moreover, one more highervoltage branch appeared, confirming the assumption that the real Swihart velocity is slightly higher than that related to the maximum measured frequency. As a further test, the lateral dimension was reduced at one side of the junction, i.e., the geometry was changed from the configuration of Fig. 4(a) to the one of Fig. 4(b). In this case the fine structure disappeared on the ZFS1 $I-V$ curve: The corresponding plot is shown in Fig. 5(b). In Figs. 5(a) and 5(b) the only accessible part of the step is shown. Further, the lower asymptotic voltage of the step in Fig. 5(b) is a well-known effect due to the reduction of the idle region. ${ }^{14,15}$

\section{THE MODEL}

A well-known property of solitons in integrable systems is that at zero dissipative losses there is no radiation coming out of a soliton-antisoliton collision, which is equivalent to a fluxon collision with an open boundary. In general, presence of dissipation leads to radiation losses (emission of plasma waves) during soliton-antisoliton collisions; ${ }^{16}$ however,
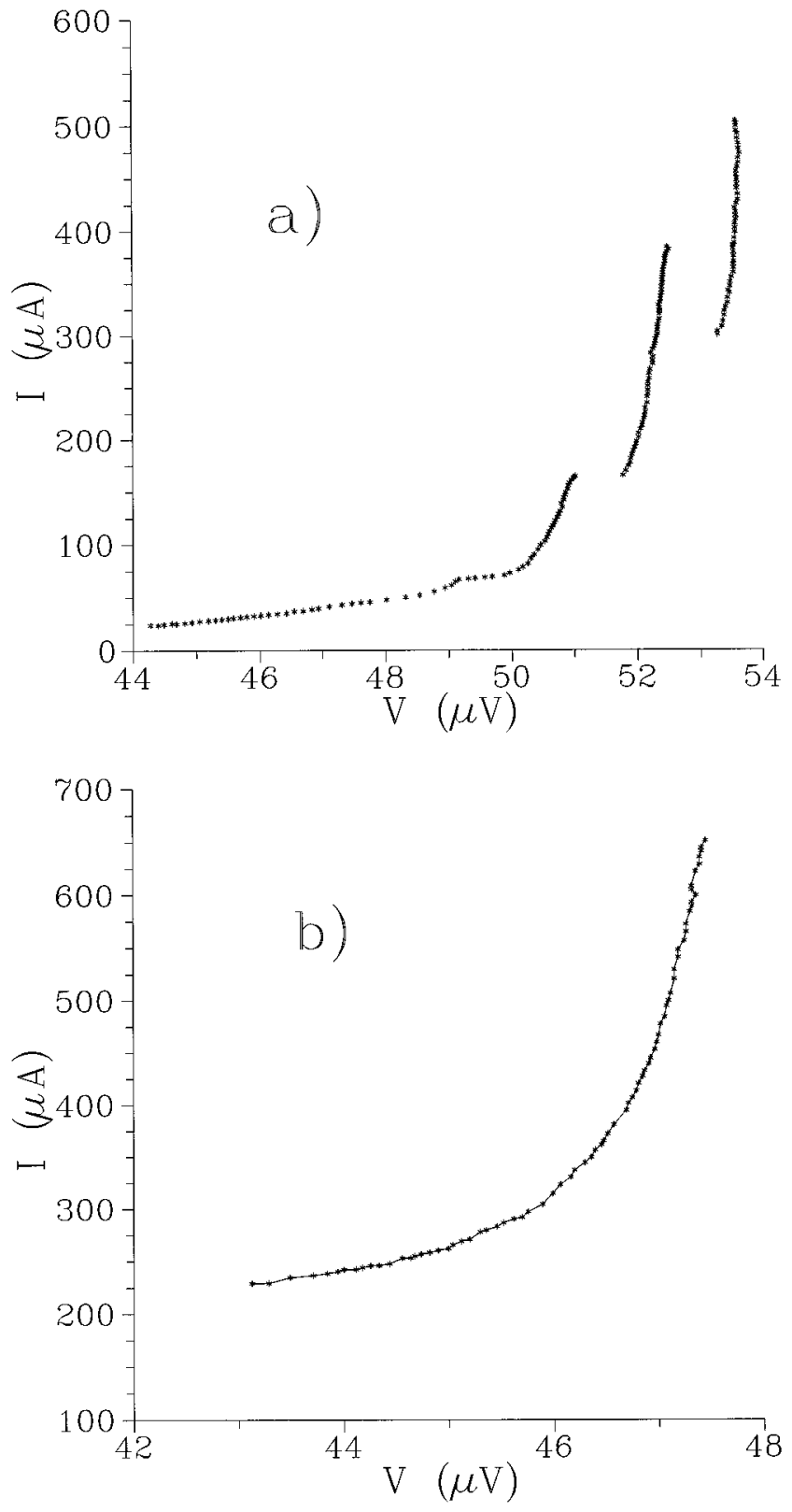

FIG. 5. $I-V$ curves of the ZFS1 of the sample A corresponding to geometries (a) and (b) of Fig. 4.

solely this effect is rather small and is usually difficult to detect in experiment. The picture drastically changes when spatial inhomogeneities are present in the system. Such inhomogeneities act as an energy potential upon the soliton and the latter usually emits radiation during its acceleration or deceleration. Strong radiation effects leading to resonances in $I-V$ curves have been found before ${ }^{8}$ both experimentally and theoretically in long junctions with periodically placed inhomogeneities. More recently, a similar theoretical approach has been successfully applied to the case of twofluxon dynamics (ZFS2) and the radiation-induced fluxon bound states. $^{17}$

If the boundaries of a junction are nonideal they may be treated as local inhomogeneities. In real experiment, the nonideality can be foreseen due to, for example, photolitho- 
graphic roughness at the junction edges. In such a case, the profile of the critical current density per unit length along the junction falls to zero near the edge not sharply, but is smeared over a certain length determined by the roughness scale. At an inhomogeneous boundary the emission of the radiation may take place. In general, the influence of the radiation on fluxon motion is not negligible if $\alpha l<1$, where $l=L / \lambda_{J}$.

Let us consider a simple kinematic model where the boundaries are the points where the fluxon radiates plasma waves. We assume that the wave number $k$ of the emitted waves is different from zero. If one fixes the normalized junction length $l$ and the average fluxon velocity $u$, it is possible to derive a condition of the interference between the waves emitted sequentially from opposite boundaries due to fluxon collisions. Such a procedure allows to calculate the fluxon spacing as a function of their velocity and takes into account plasma waves with $\omega=\sqrt{1+k^{2}}>1$. We assume that the main radiation field (in general, containing waves with different $k$ ) is excited due to fluxon collision with junction boundaries. For a fluxon moving with a certain average velocity $u$ there will be an interference between periodically emitted plasma waves with a particular wave number $k_{0}$ which depends on $u$ and $l{ }^{18}$ In its turn, depending on $u$, the temporally periodic fluxon motion may lead to a resonance ${ }^{8}$ between the traveling plasma waves with $k=k_{0}$ and the fluxon frequency $f_{f}=u /(2 l)$ itself. This resonance condition has been obtained by Golubov and Ustinov ${ }^{8}$ as

$$
u_{n}=\left[\left(1-\frac{l_{1}}{n a}\right)^{2}+\left(\frac{l_{1}}{2 \pi n}\right)^{2}\right]^{1 / 2},
$$

where $u_{n}$ are the normalized fluxon velocities at which the resonances take place. Here $n$ is an integer, $a=l$ (spacing between inhomogeneities, which are essentially the junction boundaries), and $l_{1}=2 l$ (fluxon spatial period for the ZFS1, i.e., twice the junction normalized length $l$ to take into account the linear geometry of our samples).

For this particular case, Eq. (4a) reduces to

$$
u_{n}=\left[\left(1-\frac{2}{n}\right)^{2}+\left(\frac{l}{\pi n}\right)^{2}\right]^{1 / 2} \text {. }
$$

Figure 6 shows the dependence given by Eq. (4b) (dashed line) together with the experimental positions of the fine structure steps given by open circles. Figure 6(a) and 6(b) account for two different samples A and B listed in Table I. In order to fit theory to experiment, we assumed that the true experimental Swihart velocity was somewhat higher than that corresponding to the highest voltage detected on ZFS1. Since the very top part of the step is usually unstable this assumption is quite realistic. In the fits we used the velocity correction by factor of 1.16 for sample A and 1.12 for sample B. The agreement between theory end experiments appears to be rather good: The spacing between resonances is decreasing as $u$ approaches unity, as observed in experiment. The model correctly describes the temperature dependence of the fine structure: It is quite clear that the structure should disappear at high temperatures (as it is observed in experiment) when the higher losses dump out the plasma oscillations.
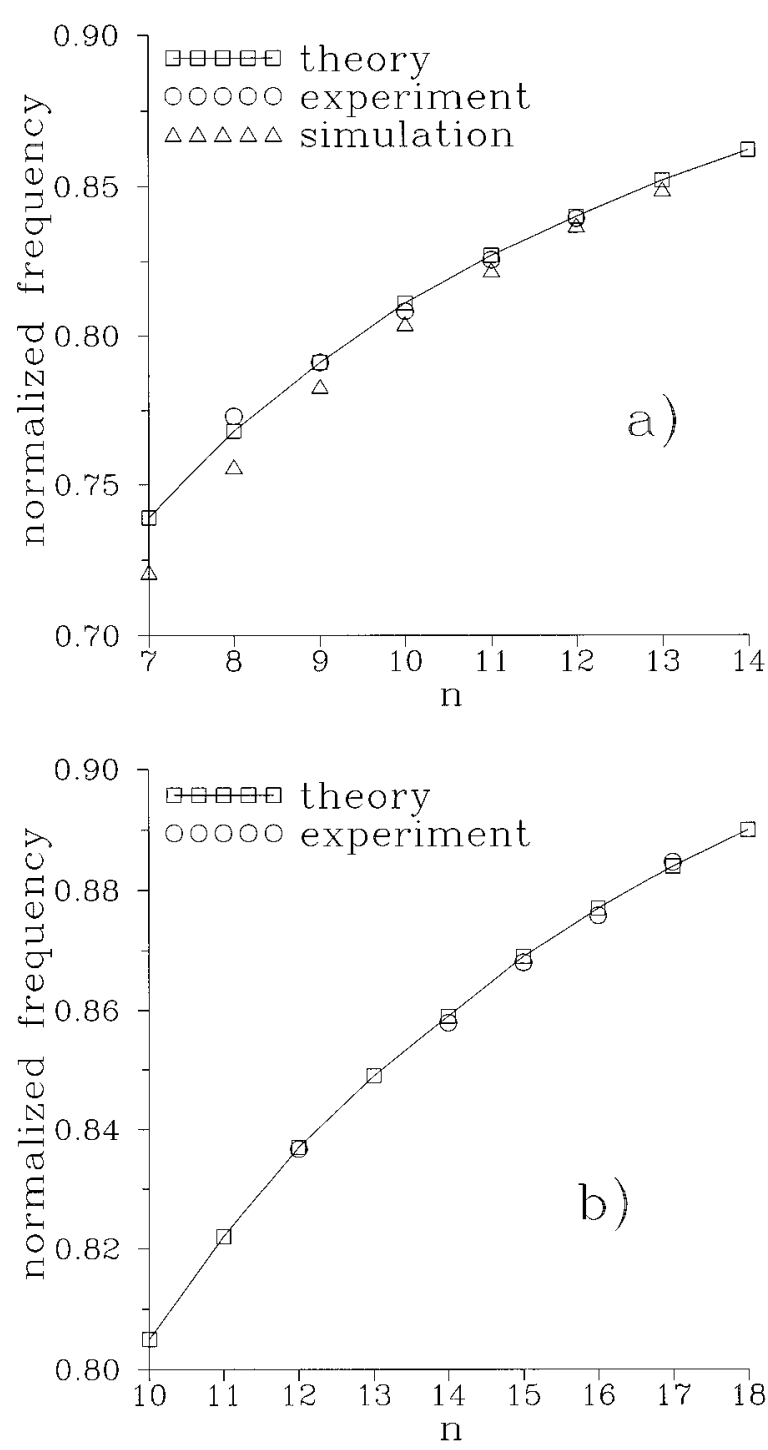

FIG. 6. Normalized fluxon velocities corresponding to the fine structure resonances as a function of the resonance number $n$. Theoretical values (open squares connected by a solid line) are given by Eq. (4b) and experimental data are reported as open circles. Cases (a) and (b) account for the samples A and B, respectively. For sample A, the results of full numerical simulations using Eq. (5) are also reported (open triangles).

Let us discuss here the experiment reported above for various dimensions of the idle region (window) between superconducting electrodes around the junction. The magneticfield lines associated with the fluxon extend into the dielectric of the idle region. When the fluxon moves near the junction edge these lines close around the edge outside the junction area, thereby producing an additional (self-induced) magnetic field at the junction boundary. Such a field configuration acts as a perturbation to the ideal open end boundary condition, due to the interaction of the fluxon with the selfgenerated magnetic field (this field has opposite polarity to the field inside the fluxon) at this boundary. ${ }^{15}$ Obviously, the fluxon motion is perturbed and one may expect radiation of plasma waves at these regions. Experimentally made reduction of the junction lateral dimension removed the fine structure. Indeed, if the area of fluxon extension into the idle 


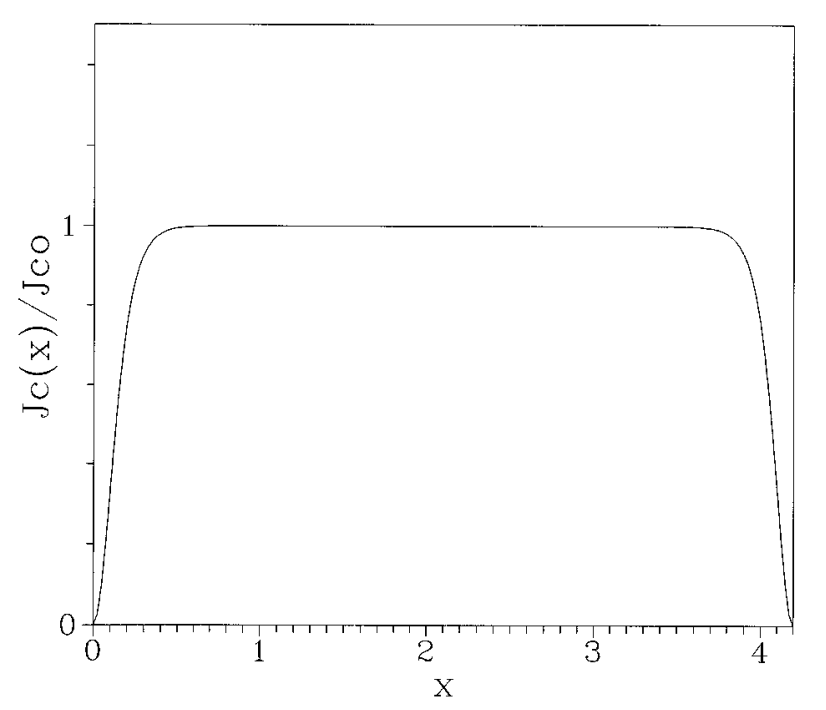

FIG. 7. The critical current density profile $g(x)$ corresponding to nonideal boundaries at $x=0$ and $x=4.2$. The normalized width of each inhomogeneous region is 0.3 .

region is small enough, no self-generated field is expected since the field lines close in the open space outside the superconducting electrodes.

In order to explore the theoretical model in greater detail we performed direct numerical simulations of the onedimensional fluxon dynamics in a junction with inhomogeneous boundaries. The simulations were performed using the perturbed sine-Gordon equation,

$$
\frac{\partial^{2} \varphi}{\partial x^{2}}-\frac{\partial^{2} \varphi}{\partial t^{2}}=g(x) \sin \varphi+\alpha \frac{\partial \varphi}{\partial t}+\eta
$$

where $g(x)$ accounts for the spatially dependent critical current density per unit length of the junction. We used the profile $g(x)$ shown in the Fig. 7, which imitates the profile of the critical current density per unit length felt by the fluxon. Simulations were performed with a set of parameters close to that of experimentally measured sample A: $l=4.2$ and $\alpha=0.01$. For simplicity, we excluded in simulations the loss term $\beta \partial^{3} \varphi /\left(\partial x^{2} \partial t\right)$. Since $\beta$ losses are responsible for the shape of the $I-V$ curve at high bias, ${ }^{13}$ we may expect an agreement with experiment only in the velocity position of the resonances (e.g., in the low-bias region), and not in the shape of the resonances themselves. $I-V$ characteristic corresponding to the ZFS1 has been calculated in conventional way using as the initial condition of the first $I-V$ point a fluxon placed in the junction and moving with a certain velocity. For each value of the bias current $\eta$, Eq. (5) was solved numerically until stationary oscillations are achieved (typically, with integration time of about 1000 time units). The bias current increment was inverted at several points of the $I-V$ curve in order to trace out various hysteretic branches. The dc voltage in simulations was normalized to be equal to the average fluxon velocity $u$.

The simulated ZFS1 is shown by solid squares in Fig. 8 in which the voltage is normalized to $\Phi_{0} \bar{c} / L$ and the bias is normalized to $I_{c}$. Thin solid line indicates the $I-V$ curve

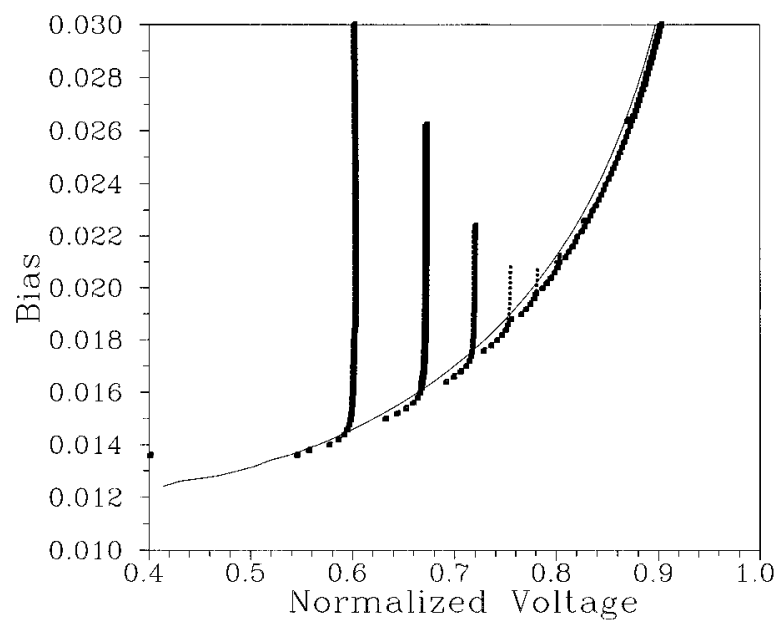

FIG. 8. Numerically simulated $I-V$ curve (solid squares) of the long Josephson junction with inhomogeneous boundaries described by Eq. (5), using the profile $g(x)$ shown in Fig. 7. The junction parameters used in the simulations are close to that of experimentally measured sample A: $l=4.2$ and $\alpha=0.01$. The solid line indicates the $I-V$ curve calculated for a homogeneous $[g(x) \equiv 1]$ junction with the same parameters $l$ and $\alpha$.

calculated for a homogeneous $[g(x) \equiv 1]$ junction with the same parameters $l$ and $\alpha$. We see that, in difference from the smooth curve of the homogeneous junction, the simulated ZFS1 for the junction with inhomogeneous boundaries $[g(x)$ given by Fig. 7] shows sharp resonant branches. The voltage positions of these branches, according to Eq. (4b), does not depend on the exact form of $g(x)$ provided it is enough peaked at the edges. The variation of the normalized width of the inhomogeneous regions of $g(x)$ from 0.2 to 0.5 and the depth from 0.5 to 1.0 showed very minor influence on the voltage positions of the branches but varied only their current amplitude.

A detailed numerical inspection of the temporal voltage dependence in any point inside the junction allows to determine the number of plasma oscillations $n$ [see Eq. (4b)] per one fluxon oscillation period. Indeed, this number $n$ is found to be different for different resonant branches shown in Fig. 8 . The corresponding dependence of the numerically calculated resonance velocities on the resonance number $n$ is shown in Fig. 6(a) together with experimental data and analytically predicted points. One can see good agreement between experiment, theory, and numerical simulations.

Since our model involves traveling plasma waves which do not coincide with cavity modes of the junction, we do not expect that these waves can be directly detected in experiment using time-averaging spatially resolved techniques such as low-temperature scanning electron microscopy (LTSEM). An obvious way to observe these waves is to perform radiation measurements at the $n$th harmonic of the fluxon frequency.

\section{CONCLUSION}

The fine structures often observed in the first zero field steps are ascribed to the resonance between the fluxon and plasma waves generated during its motion. The crucial factor for the occurrence of fluxon-plasma wave resonances is the 
presence of spatially inhomogeneous regions in the junction which perturb the fluxon motion. In other words, plasma waves are generated when the fluxon is accelerated at inhomogeneities. The extremities of a linear junction may act as inhomogeneities either when the bias current is injected into the junction at the edges as in the case of very long junctions or when the junction is surrounded by a large idle region, in the latter case independently of its electrical length. In this work, we did not consider the inhomogeneity of the bias current because our samples were intermediate length junctions for which the critical current scales with the junction area. We present a model which properly describes all the experimental features, including the temperature dependence of the fine structure. The model takes into account nonideal boundaries of the junction as presented above. We also performed full numerical simulations of this problem and found good agreement between theory and experiment.

\section{ACKNOWLEDGMENTS}

The authors wish to thank Professor G. Costabile and Professor R. D. Parmentier for stimulating discussions and for a critical reading of the manuscript; further we acknowledge A. Ferrentino for valuable technical assistance. This work was partially supported by the Consiglio Nazionale delle Ricerche under the Progetto Finalizzato "Superconductive and Cryogenic Technologies," by the European Union under the "Science" program and by the MURST (Italy).
${ }^{1}$ D. W. McLaughlin and A. C. Scott, Phys. Rev. A 18, 1652 (1978).

${ }^{2}$ A. Davidson, B. Dueholm, B. Kryger, and N. F. Pedersen, Phys. Rev. Lett. 55, 2059 (1985).

${ }^{3}$ M. R. Scheuermann, T. V. Rajeevakumar, J. J. Chang, and J. T. Chen, Physica B 107, 543 (1981).

${ }^{4}$ J. J. Chang, J. T. Chen, M. R. Scheuermann, and D. J. Scalapino, Phys. Rev. B 31, 1658 (1985).

${ }^{5}$ R. Monaco, P. Barbara, and J. Mygind, Phys. Rev. B 47, 12292 (1993).

${ }^{6}$ M. R. Scheuermann, C. C. Chi, N. F. Pedersen, J. J. Chang, and J. T. Chen, Appl. Phys. Lett. 48, 189 (1986).

${ }^{7}$ S. N. Erne' and R. D. Parmentier, J. Appl. Phys. 51, 5025 (1980).

${ }^{8}$ A. A. Golubov and A. V. Ustinov, IEEE Trans. Magn. MAG-23, 781 (1987); A. A. Golubov, I. L. Serpuchenko, and A. V. Ustinov, Zh. Eksp. Teor. Fiz. 94, 297 (1988) [Sov. Phys. JETP 67, 1256 (1988)].

${ }^{9}$ H. Kroger, L. N. Smith, and D. W. Jillie, Appl. Phys. Lett. 39, 280 (1981).

${ }^{10}$ R. Monaco, R. Cristiano, L. Frunzio, and C. Nappi, J. Appl. Phys. 71, 1888 (1992).

${ }^{11}$ E. Jorgensen, V. P. Koshelets, R. Monaco, J. Mygind, M. R. Samuelsen, and M. Salerno, Phys. Rev. Lett. 49, 1093 (1982).

${ }^{12}$ E. Jorgensen, Ph.D. thesis, The Technical University of Denmark, 1983.

${ }^{13}$ S. Pagano, M. P. Soerensen, P. L. Christiansen, and R. D. Parmentier, Phys. Rev. B 38, 4677 (1988).

${ }^{14}$ R. Monaco, G. Costabile, and N. Martucciello, J. Appl. Phys. 77, 1888 (1995).

${ }^{15}$ N. Thyssen, A. V. Ustinov, H. Kohlstedt, S. Pagano, J. G. Caputo, and N. Flytzanis, IEEE Trans. Appl. Supercond. AS-5, 2965 (1995).

${ }^{16}$ Yu. S. Kivshar and B. A. Malomed, Zh. Exp. Teor. Fiz. 90, 2162 (1986) [Sov. Phys. JETP 63, 1267 (1986)].

${ }^{17}$ S. G. Lachenmann, G. Filatrella, A. V. Ustinov, T. Doderer, N. Kirchmann, D. Quenter, R. P. Huebener, J. Niemeyer, and R. Pöpel, J. Appl. Phys. 77, 2598 (1995).

${ }^{18}$ G. S. Mkrtchyan and V. V. Schmidt, Solid State Commun. 30, 791 (1979). 